Boise State University

ScholarWorks

$4-2020$

Fort Mcdermitt Reservation (Mcdermitt, Nevada)

Tim Thornes

Boise State University

This document was originally published in International Journal of American Linguistics by University of Chicago Press. Copyright restrictions may apply. doi: 10.1086/707229 


\title{
FORT MCDERMITT RESERVATION (McDERMITT, NEVADA)
}

\author{
Tim THORNeS
}

Boise State University

The McDermitt reservation straddles the Oregon-Nevada border and is the home of the majority of all remaining first language Northern Paiute speakers. Like the Duck Valley Reservation, it is quite isolated, lying approximately 90 miles northeast of the town of Winnemucca, Nevada (population $>7,000$ ). The following stories are two of the many texts collected in the early 1960s by Sven Liljeblad from Pete Snapp, a fluent speaker from McDermitt. Mr. Snapp was in his early nineties at the time of this recording, which was made on reel-to-reel tape and archived as part of the Sven Liljeblad Collection in the Special Collections Department at the University of Nevada at Reno. His interlocutor was more than likely his son, Allen Snapp, who worked with the missionaries John and Joy Anderson to produce the first fairly complete grammar sketch of Northern Paiute (Snapp et al. 1982).

\section{THE BEDWETTER STORY ${ }^{1}$}

Pete SnAPP, NARRATOR

The "Pete Snapp Tales" archived in the Special Collections Department at the University of Nevada at Reno include numerous narratives that might be considered traditional rather than historical. As with many oral narratives from the time period, "The Bedwetter Story" presented here combines elements of both types, blurring the line that distinguishes the two.

Liljeblad developed the system of transcription shown in the first line of text. In it, the vowels carry their standard Latin values, with the addition of $<y>$ for the high central vowel $/ \mathrm{i} /$. In the Americanist tradition, the alveolar and alveopalatal afficates are represented as $\langle\mathrm{c}\rangle$ and $<\breve{\mathrm{c}}\rangle$, respectively, and the alveopalatal fricative as $\langle\check{\mathrm{s}}>$. For the palatal glide, Liljeblad uses $<j>$. His representation of proclitics of various sorts (possessor and object pronominals in particular) as part of the word necessitates the marking of primary stress, for which an acute accent $<^{\prime}>$ is used. This approach masks the highly predictable, second mora, primary stress system of the language. Knowledge of that system makes identifying proclitics a fairly simple matter, since proclitics, unlike prefixes, do not trigger leftward stress shift (see the introduction to this supplement).

${ }^{1}$ Recorded by Sven Liljeblad in 1962, Fort McDermitt Indian Reservation. 
Final features and consonant gradation, active earmarks of every Numic language, are indicated cleverly in Liljeblad's system, making it possible to derive a fairly accurate pronunciation of forms. An apostrophe $<$ ' $>$ in front of a consonant indicates the pronunciation as fortis (or geminate). In Pete Snapp's speech, as a speaker of one of the northern dialects, such consonants are pronounced with a longer closure and, if obstruent, voiceless. In the default case (i.e., without the preceding apostrophe), the pronunciation has been referred to as lenis, capturing a cluster of phonetic features in the production of the consonant, including voicing and frication in word medial position and a lack of aspiration word-initially.

(1) Ahaa nyy kanaa'ci ynyy na'siity naatykwina'kwai'ty natykwina'ku.

aha nii ka=naatsi ini na-sii-di

yes I ACC=boy INT MID-urinate-NMLZ

nadigwina-kwai-ti nadigwina-ku

bed-AREA-LOC bed-LOC

'Yes, I will tell the story about the boy who wet his bed all the time.'

(2) u'my ku'cu'ty'ka kajai'si unau nopi'ka?ju.

ummi kutsu-tika ka=yaisi una-u nobi-ka?yu

they cattle-eat ACC=then out.there-FOC house-have

'The people living there were cattle eaters.'

(3) unau nopi'kaana, my'papí?ikaa waahu patymyka?ju

una-u nobi-kaa-na mi=pabi?i-gaa

out.there-FOC house-have-PTCP $\mathrm{PL}=$ elder.brother-have

waa-hu padi-mi-ga?yu

two-ACC daughter-PL-have

'They lived somewhere, and their chief had two daughters.'

(4) tuPi hakatui u'katúu'kwani'i kwi'si'ky'ti nami'ku mynotý'kwakaa'ku miikaa na?yna'kwi, u'su my'papí?i miu yna'kwi.

tu $\mathrm{Pi}=$ haga $=$ tui $\mathrm{u}-\mathrm{ka} \quad$ tuu-kwayi?i kwissi-ki-ti

any $=$ who $=$ any $3 \mathrm{SG}-\mathrm{ACC}$ black-fox capture-APPL-TNS

nami-ku $\mathrm{mi}=$ nodikwa-gaa-ku $\mathrm{mii}=\mathrm{kaa}$

both-ACC PL=wife-have-ACC QUOT=thusly

na-Pinakwi u-su mi=pabí?i mi-u ina'kwi

MID-claim 3SG-NOM PL=elder.brother QUOT-FOC claim

" "Whoever catches the black fox shall have both for his wives," he said, (and thus) it was made known what the chief proclaimed.'

(5) mynymýjai'si katuu'kwayi utuihy?a.

$\mathrm{mi}=$ nimi $=$ yaisi $\quad \mathrm{ka}=$ tuu-kwayi?i $\mathrm{u}=$ tui-hi?a

$\mathrm{PL}=$ person $=$ then $\quad \mathrm{ACC}=$ black - fox $3 \mathrm{SG}: \mathrm{ACC}=$ try-trap

'The people then tried to trap the black fox.' 
(6) utuihyana kai uwy'sî'ky'ti.

$$
\begin{array}{lll}
\mathrm{u}=\text { tui-hia-na } & \text { kai } & \mathrm{u}=\text { wissí-ki-ti } \\
\text { 3SG:ACC=try-trap-PTCP } & \text { NEG } & \text { 3SG:ACC=capture-APPL-TNS }
\end{array}
$$

'They tried to trap him, but they could not catch him.'

(7) u'su ynyy na'siity naa'cika ka'tyhu'cíinoo unau nopi'ka?ju.

$$
\begin{aligned}
& \text { u-su ini na-sii-di naatsi=ga } \\
& \text { 3SG-NOM INT MID-urinate-NMLZ boy }=\text { MOD } \\
& \mathrm{ka}=\mathrm{ti}=\text { hutsi?i-noo una-u nobi-kaPyu } \\
& \mathrm{ACC}=\mathrm{LOGO}=\text { grandmother }-\mathrm{COM} \text { out there-FOC house-have }
\end{aligned}
$$

\begin{tabular}{|c|c|c|}
\hline $\mathrm{u}-\mathrm{su}$ & $\mathrm{ka}=$ yaisi & $\mathrm{ka}=\mathrm{ti}=$ hutsí?i \\
\hline $\begin{array}{l}\text { 3SG-NOM } \\
\text { nitamma }\end{array}$ & $\begin{array}{c}\mathrm{ACC}=\text { then } \mathrm{QUOT}-\mathrm{FOC} \\
\ddot{\mathrm{i}}=\text { sakwa }\end{array}$ & $\begin{array}{l}\mathrm{ACC}=\mathrm{LOGO}=\text { grandmother } \\
\mathrm{i}=\text { hutsí?i }\end{array}$ \\
\hline tell & $\mathrm{you}=\mathrm{MOD}$ & 1SG.POSS = grandmother \\
\hline manati & him & $i=v$ \\
\hline some & what & $1 \mathrm{SG}: \mathrm{ACC}=$ trap - make $-\mathrm{APF}$ \\
\hline nii & $\mathrm{u}=\mathrm{hia}-\mathrm{ku}$ & $\mathrm{mii}=$ \\
\hline I & $3 \mathrm{SG}: \mathrm{ACC}=\operatorname{tr}$ & QUOT $=$ thusly \\
\hline
\end{tabular}

'The boy who always wet his bed lived there with his father's mother.'

(8) u'sukajai'si miu ka'tyhu'cí?i ni’ta'ma yy'sa'kwa, ihu'cí?i mana'ty hi'maa iwanátu'ky [nyy u?] nyy uhya'ku miika.

'And so he said to his father's mother: "My grandmother, you should manage to make me a trap of some kind, so I can catch him," he said.'

(9) hi'ma kai ukwi'sí'kywa?ni?ju, nymy pisa?jua'pa kai ukwi'sí'ky'ti nanana uhya' paana jai'si sa'kwa?ýy hau mani miika uni’táma.

$$
\begin{aligned}
& \text { himma kai } \mathrm{u}=\text { kwissi-ki-wa?ni-Pyu nimi } \\
& \text { what NEG 3SG:ACC =capture-APPL-SIMIL-NOM person } \\
& \text { pisa-Pyu =apa kai } \mathrm{u}=\mathrm{kwissi}-\mathrm{ki}-\mathrm{ti} \\
& \text { good-NOM=MOD NEG 3SG:ACC =capture-APPL-TNS } \\
& \text { na nana } \quad \mathrm{u}=\text { hia' }- \text { paana } \quad \text { yaisi }=\text { sakwa }=\text { ?ii } \\
& \mathrm{PL} \sim \text { man } 3 \mathrm{SG} \text { :ACC }=\text { trap }-\mathrm{PROH} \text { then }=\mathrm{MOD}=\text { you } \\
& \text { hau mani } \mathrm{mii}=\mathrm{ka} \quad \mathrm{u}=\text { nittama } \\
& \text { how do QUOT=thusly 3SG:ACC=tell }
\end{aligned}
$$

" "Of course you cannot catch him; even good people cannot catch him, the men who tried to trap him; and what should you then do," she told him.'

(10) sunaa'cikaa uninóojy'kwi.

$\mathrm{su}=$ naatsi $=$ ga $\quad \mathrm{u}=$ ni-noo-yikwi

$\mathrm{NOM}=$ boy $=$ MOD $3 \mathrm{SG}: \mathrm{ACC}=\mathrm{IP} / \mathrm{SPEECH}-$ carry $-\mathrm{HAB}$

'But the boy continued to beg her.' 
(11) ynyyti'tui iwanátu'ky miika.

initi $=$ tui $\mathrm{i}=$ wana-tu-ki $\quad \mathrm{mii}=\mathrm{ka}$

$\mathrm{INT}=$ any $\quad 1 \mathrm{SG}: \mathrm{ACC}=$ trap-make-APPL $\quad$ QUOT $=$ thusly

“"Go ahead and make me a trap," he said.'

(12) uhu'cíikajai'si (ka?), ka'ty'kawónoma'ty kauwy'kwýy'ky ú'ni'ku hi'ma kwa'sy'py kawónokaana.

$\mathrm{u}=$ hutsii $\quad \mathrm{ka}=$ yaisi $\mathrm{ka}=\mathrm{ti}=$ kawono-mati

3SG.POSS $=$ pat.grandmother $\quad \mathrm{ACC}=$ then $\quad \mathrm{ACC}=\mathrm{LOGO}=$ basket $-\mathrm{PART}$ $\mathrm{ka}=\mathrm{u}=$ wi-kwii-ki uuni-ku himma

$\mathrm{ACC}=3 \mathrm{SG}: \mathrm{ACC}=\mathrm{IP} / \mathrm{LONG}-$ grasp-APPL that.kind-ACC what

kwassipi kawono-kaa-na

buckskin burden.basket-have-PTCP

'His father's mother then cut part of her kawono into strips; it was that kind, some kind of tanned buckskin she had for a kawono. ${ }^{2}$

(13) jai'si, sunaa'ci unaa uhyatia?a katuukwani'i uhya'tua.

yaisi $\mathrm{su}=$ naatsi una $\mathrm{u}=\mathrm{hia}=$ tia?a

then $\mathrm{NOM}=$ boy out.there $3 \mathrm{SG}: \mathrm{ACC}=$ trap $=$ thusly

$\mathrm{ka}=$ tuu-kwani?i $\mathrm{u}=$ hia-tua

$\mathrm{ACC}=$ black-fox $\quad 3 \mathrm{SG}: \mathrm{ACC}=$ trap-IRR

'And then ... the boy somewhere set a trap for him, set a trap for the black fox.'

(14) sunaa'cijai'si ukwi'sí'kyu.

$\mathrm{su}=$ naatsi $=$ yaisi $\mathrm{u}=\mathrm{kwissi}-\mathrm{ki}-\mathrm{u}$

$\mathrm{NOM}=$ boy $=$ then $3 \mathrm{SG}: \mathrm{ACC}=$ capture $-\mathrm{APPL}-\mathrm{PFV}$

'And the boy caught him.'

(15) ukwi'sî'kyu sunaa'ci?i oo mí?a'su.

$\mathrm{u}=\mathrm{kwissi}-\mathrm{ki}-\mathrm{u} \quad \mathrm{su}=$ naatsi?i oo mú $\mathrm{Pasu}$

3SG:ACC = capture-APPL-PFV NOM=boy DEM already

'There the boy had already caught him.'

(16) [u'my a'papí?i] a'papíi 'patymy nami'ku mynotý'kwatu, nazatimitia?a.

ummi a=pabi?i a=pabi?i padi-mi

they $4=$ elder.brother $4=$ elder.brother daughter-PL

nami-ku $\mathrm{mi}=$ nodikwa-tu na-dzatimi $=$ tia?a

both-ACC $\mathrm{PL}=$ wife-make $\quad$ MID-provide $=$ thusly

'The chief's daughters, both of them, he married; they were given (to him).'

\footnotetext{
${ }^{2}$ A conical burden basket used for gathering (and carrying) berries, usually coarsely woven, but with buckskin sometimes used as straps for carrying over the shoulders.'
} 
(17) u'myjai'si pu'kwa'kaa.

ummi=yaisi pokwa-kaa

they $=$ then lie:PL-away

'And they went to bed.'

(18) sunaa'ci mýnoo hapi'kaa.

$\mathrm{su}=$ naatsi mi-noo hapi-kaa

NOM=boy PL-COM lie:SG-away

Listener: 'Did the boy sleep with them?'

(19) sunaa'ci mýnoo hapi'kaa.

$\mathrm{su}=$ naatsi mi-noo hapi-kaa

NOM=boy PL-COM lie:SG-away

'The boy slept with them.'

(20) u'mynoo hapi'kaa'si jai'si u'mykupa'tu na'sii.

ummi-noo hapi-kaa-si yaisi

they-COM lie:SG-away-SEQ then

ummi-kupa-tu na-sii

they-upon-to MID-urinate

'And when he slept with them, he urinated on them.'

(21) u'myjai'si, suha'máakajai'si kau'sunáa'pynoo'koso u'na' pýywa'na'tu upiwó?ja'kwynaihu.

ummi =yaisi $\quad \mathrm{su}=$ hamma?a $\quad \mathrm{ka}=$ yaisi

they $=$ then $\quad \mathrm{NOM}=$ elder.sister $\quad \mathrm{ACC}=$ then

$\mathrm{ka}=\mathrm{u}=$ sunaapi nookoso una

$\mathrm{ACC}=3 \mathrm{SG} . \mathrm{POSS}=$ bedding everything out.there

pïwana-tu $\mathrm{u}=$ biwó $y$ ya-kwinai-hu

outdoors-to 3sG:ACC $=$ drag-throw:SG-PFV

'They then, the older sister then drove him outside with his bedding.'

(22) jaato siina mau ma?na?wi mi uni'támana.

$\begin{array}{llll}\text { yaa-to } & \text { sii-na } & \text { ma-u } & \text { ma?na?wi } \\ \text { here-LOC } & \text { urinate-PTCP } & \text { DEF-FOC } & \text { do/act } \\ \text { mii } & \mathrm{u}=\text { nittama-na } & & \\ \text { QUOT } & \text { 3SG:ACC=tell-PTCP } & \end{array}$

“"Piss here that is what you can do," she told him.'

(23) uu'sa'pa jai'si naku'cumai'si, nymy u'mykuba?juna nomiča.

$\mathrm{uu}=$ sapa $\quad$ yaisi na-kutsu-mai-si nimi

like.so $=$ MOD then MID-cow-do-SEQ person

ummi-guba-Pyu-na nomiča

they-upon-from-PTCP move.away

'And then it happened, when the cattle were herded, that the people moved away from them.' 
(24) u'su u'py'núikaa kai mi?a u'mypaa?juna.

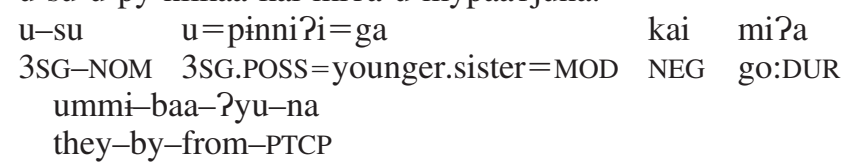

'The younger sister did not go from them.'

(25) u'su u’py'níikaa oo kuhu'cíipaa'sutia?a.

$$
\begin{aligned}
& \mathrm{u}-\mathrm{su} \quad \mathrm{u}=\text { pinni?i=gaa } \quad \text { oo } \\
& \text { 3SG-NOM 3SG.POSS }=\text { younger } . \text { sister }=\text { MOD DEM } \\
& \mathrm{ka}=\text { hutsi } \mathrm{Pi}-\mathrm{baa}-\mathrm{su}=\text { tia?a } \\
& \mathrm{ACC}=\text { grandmother }- \text { by }-\mathrm{ADV}=\text { thusly }
\end{aligned}
$$

'The younger sister remained there with his father's mother.'

(26) sunaa'citia ka?oono jakana miu y?na?wi'si, una kuta'kwama'tu mi?a.

$\begin{array}{clll}\mathrm{su}=\text { naatsi }=\text { tia } & \mathrm{ka}=\text { Poono } & \text { yaka-na mi-u } \\ \text { NOM=boy=thusly } & \mathrm{ACC}=\text { at.the.time } & \text { cry-PTCP } & \text { QUOT-FOC } \\ \text { i?na?wi-si } & \text { una } & \text { kudakwa-ma-tu } & \text { mi?a } \\ \text { talk-SEQ } & \text { out.there } & \text { butte-LOC-to } & \text { go:DUR }\end{array}$

'The boy again, as he was crying there and talking (by himself), went over to the butte.'

(27) oo'kaa napiwo?ja'kwynaiu'si.

$\mathrm{oo}=\mathrm{kaa} \quad$ na-biwo?ya-kwinai-u-si

like.so=MOD MID-drag-throw:SG-PFV-SEQ

Listener: 'Was that when he was dragged out?'

(28) oo'kaa napiwo?ja'kwynaiu'si.

$\mathrm{oo}=\mathrm{kaa} \quad$ na-biwo?ya-kwinai-u-si

like. so $=$ MOD MID-drag-throw:SG-PFV-SEQ

'That was when he was dragged out.'

(29) una?ju Pnajai'si sunaa'ci, jakana miu y?na?wi.

una-Pyu-Pna=yaisi $\quad$ su=naatsi yaka-na $\mathrm{mi}-\mathrm{u}$

out.there-from-PTCP $=$ then $\quad \mathrm{NOM}=$ boy $\quad$ cry-PTCP $\quad$ QUOT-FOC

i?na?wi

talk

'Somewhere over there the boy was crying and talking.'

(30) jakana miu y?na?wina joyo'kaa'si.

yaka-na mi-u i?na?wi-na yojo-kaa-si

cry-PTCP QUOT-FOC talk-PTCP evening-away-SEQ

'He was crying and talking until it got late in the evening.' 
(31) sutuihíi'tui'kaa upaa pi'ty?ja'kwi pisa?jutia?a hii'pi.

$$
\begin{aligned}
& \mathrm{su}=\text { tui }=\text { hii }=\text { tui }=\mathrm{kaa} \quad \mathrm{u}-\text { baa pitti-?yakwi } \\
& \mathrm{NOM}=\text { any }=\text { thing }=\text { any }=\mathrm{MOD} \quad 3 \mathrm{SG}-\text { by } \quad \text { arrive }-\mathrm{HAB} \\
& \text { pisa-?yu }=\text { tia?a hiipi } \\
& \text { good-NOM=thusly whatever }
\end{aligned}
$$

'Then anything that could be (regarded as) property came to him, anything that was good to be owned.'

(32) tuihíitui, paka'pytia?a, pisa?ju waa?aty, upaa mani'mina wika hau'ni?ju.

tui $=$ hii $=$ tui $\quad$ pagapi $=$ tia?a pisa - Pyu $\quad$ waa - Padi

any $=$ thing $=$ any arrow $=$ thusly good-NOM juniper-bow

u-baa mani-mina wiga hau'ni-?yu

3SG-by do-go.along rabbit.blanket some.kind-NOM

'Just anything, arrows for one thing, and good juniper bows were piling up around him, blankets, all kinds of things.'

(33) jai'sitúu, ku'cu tywaukaa, pu'ku tywau.

yaisi tuu kutsu tiwau=gaa puku tiwau

then also cattle also $=$ MOD horse also

'And even cattle and horses too.'

(34) ú'ni?jukaa upaa nymytua.

uuni-Pyu = gaa u-baa nimi-tua

that.kind-NOM=MOD 3SG-by person-IRR

'Things like that appeared by him.'

(35) sunaa'cikajai'si awamu Pa tynopí'tami umahý'ki.

$\mathrm{su}=$ naatsi $\mathrm{ka}=$ yaisi awamu $\mathrm{Pa} \mathrm{t} i=$ nobi-tami

$\mathrm{NOM}=$ boy $\quad \mathrm{ACC}=$ then morning $\quad \mathrm{LOGO}=$ house-to

$\mathrm{u}=$ mahi-ki

3SG:ACC $=$ herd-toward

'And then early in the morning the boy drove them toward his home.'

(36) katyhi'má.

$\mathrm{ka}=\mathrm{ti}=$ himma

$\mathrm{ACC}=\mathrm{LOGO}=$ what

Listener: 'His belongings?'

(37) katyhi'má oo py’paa manity.

$\mathrm{ka}=\mathrm{t} \mathrm{i}=$ himma $\quad$ oo pi-paa mani-di

ACC $=$ LOGO $=$ what DEM RESTR-by do-NMLZ

'Anything that gathered there by him.' 
(38) u'su uhu'cíika ka'syáty'mynoo ói?juna, awamu Pa kamiu, hii nyjyjyiwa'ny miika.

$$
\begin{aligned}
& \mathrm{u}-\mathrm{su} \quad \mathrm{u}=\text { hutsi } \mathrm{i}=\mathrm{ga} \quad \mathrm{ka}=\text { siadimi-noo } \\
& \text { 3SG }-\mathrm{NOM} \quad 3 \mathrm{SG} . \mathrm{POSS}=\text { grandmother }=\mathrm{MOD} \quad \mathrm{ACC}=\text { girl }-\mathrm{COM} \\
& \text { oi-Pyu-na awamu } 3 a \quad k a=m i i-u \\
& \text { DEM-NOM-PTCP morning ACC }=\text { QUOT }- \text { FOC } \\
& \text { hii niyiyï-wini } \mathrm{mii}=\mathrm{ga} \\
& \text { thing noise-PROG:SG QUOT=MOD }
\end{aligned}
$$

'His father's mother and the young girl who were there said early in the morning: "What is it that makes such noise?",

(39) susyáty'myjai'si tymua'py.

$\mathrm{su}=$ siadimi $=$ yaisi timuapi

$\mathrm{NOM}=$ girl $=$ then $\quad$ peep

'The young woman peeped out.'

(40) tymuapynajai'si, pu'ku ku'cu čaa namahyki'na iwa?ju miika.

timuapi-na=yaisi puku kutsu i-ča

peeped-PTCP $=$ then horse cow PROX-ACC

$$
\begin{array}{lll}
\text { na-mahi-ki-na } & \text { iwa-Pyu } & \text { mii=ga } \\
\text { MID-herd-toward-PTCP } & \text { many-NOM } & \text { QUOT=thusly }
\end{array}
$$

'She looked out and said: "There is a lot of horses and cows being driven this way.",

(41) uu'sa'pa oo, oo sunaa'ci mymahý'pi'ty.

$$
\begin{array}{lllll}
\mathrm{uu}=\text { sapa } & \text { oo } & \text { oo } & \text { su=naatsi } & \mathrm{mi}=\text { mahi-pitti } \\
\text { like.so=MOD } & \text { DEM } & \text { DEM } & \mathrm{NOM}=\text { boy } & \mathrm{PL}=\text { herd-arrive }
\end{array}
$$

'There the boy came driving them along.'

(42) óčai'si nopi'kaana, typa'ca'sitia u'my utykána.

$$
\begin{array}{cll}
\text { oo=čaisi } & \text { nobi-kaa-na } & \text { ti-patsa-si=tia } \\
\text { DEM=then } & \text { house-have-PTCP } & \text { ANTIP-kill:SG-SEQ=thusly } \\
\text { ummi } & \mathrm{u}=\text { tika-na } & \\
\text { they } & \text { 3SG:ACC=eat-PTCP }
\end{array}
$$

'And there they were at home, and after they made a kill, they ate it.'

(43) u'my ku'cú'maimootyčai'si pi'tytia mynymýpaa kočy'pi'ty.

$$
\text { ummi kutsu-mai-moo-di=čaisi pitti=tia }
$$

they $\quad$ cow-do-RNDM:PL-NMLZ $=$ then arrive $=$ thusly

$\mathrm{mi}=$ nimi-baa koči-pitti

$\mathrm{PL}=$ person-by return-arrive

'And then those who herded cattle came and returned to the people.'

(44) u'mykajai'si sučahítia una'ká.

ummi ka=yaisi $\mathrm{su}=$ čahi=tia $\quad \mathrm{u}=$ nakka

they $\quad \mathrm{ACC}=$ then $\mathrm{NOM}=$ father.in.law =thusly $3 \mathrm{SG}: \mathrm{ACC}=$ hear:DUR

'And they and his father-in-law heard about it.' 
(45) uPuu mani'py u'suu miika.

u Puu mani-pi u-su-u mii=ga

like.so do/happen-PERF 3SG-NOM-FOC QUOT=thusly

"'So, that is what happened to him," he said.'

(46) u'myjai'si ta'mi jau u'mypaa pi'tyukaa'ku miitia sučahí.

ummi yaisi tammi ya-u umi-baa

they then we:INCL here-FOC they-by

piti-u-gaa-ku $\quad \mathrm{mii}=$ dia $\quad \mathrm{su}=$ čahi

arrive-PFV-away-FUT QUOT=thusly $\mathrm{NOM}=$ father.in.law

'And they then, . . . "Here we will go and stay with them," his father-in-law said.'

(47) kamynáa'cipaa.

$\mathrm{ka}=\mathrm{mi}=$ naatsi-baa

$\mathrm{ACC}=\mathrm{PL}=$ boy-by

Listener: 'With the boy's family?'

(48) y'mypaa.

immi-baa

they:ACC-by

'With them.'

(49) u'mypaa pi'tyu'si jai'si ka?u'sú u'nanápypaty, upaa tuPi'pi'tyu.

ummi-baa pitti-u-si yaisi ka u-su

them-by arrive-PFV-SEQ then and 3SG-NOM

$\mathrm{u}=$ na-naa-pi-badi u-baa tu Pi-pitti-u

3SG:ACC $=$ MID-grow-PERF-daughter 3SG-by try-arrive-PFV

'They came to them, and she, his oldest daughter, tried to come to him.'

(50) oo'ty hi'matia mapi'capiwynyna, hau yy ynyy i'ka sosi pisa ha'ni miika ynina ka'ty'py'núi ni’tamana, pyy oi namapita'kwapaawynyna.

$$
\begin{aligned}
& \text { oo-ti himma=tia mabitsabi-wini-na hau it } \\
& \text { DEM-LOC what=thusly arrange-PROG:SG-PTCP how you } \\
& \text { ini i-ka sosi pisa hanni } \\
& \text { INT PROX-ACC froth good prepare } \\
& \text { mii=ga ini-na } \text { ka=ti=pinni?i } \\
& \text { QUOT=thusly say-PTCP ACC=LOGO=sis } \\
& \text { nittama-na pii } \quad \text { oi na-mabidakwabaa-wini-na } \\
& \text { tell-PTCP RESTR DEM MID-obstruct-PROG:SG-PTCP }
\end{aligned}
$$

'There she was busy putting things in order, and she said: "Why don't you take care this sosí?" So she told her younger sister, while she herself was in their way.' 
(51) oo hii'tu susosí?

oo hii-tu $\mathrm{su}=$ sosi

DEM thing-to $\mathrm{NOM}=$ froth

Listener: 'What is that sosî?'

(52) oo'su a'tyká'mawyny'kupa'kwai'ty saa, a'poo'siwyny'na.

$\mathrm{oo}=$ su $\quad \mathrm{a}=$ tika-ma-wini-kuba-kwai-ti saa

DEM=EMPH $4=$ eat-on-stand:SG-upon-AREA-LOC boiling

$\mathrm{a}=$ poosi-wini-nna

4=bubble-stand:SG-PTCP

'That is the thing that gathers on top of the food, when it is boiling, the bubbles that are coming to the top of it.'

(53) u'suu miu nani?a a'sosí mi.

u-su-u mi-u na-ni?a a=sosi mii

3SG-NOM-FOC QUOT-FOC MID-call 4=froth QUOT

'That is what is called its sosí.'

(54) u'kau wy'kitana.

$\mathrm{u}-\mathrm{ka}-\mathrm{u} \quad$ wikida-na

3SG-ACC-FOC skim-PTCP

'She was skimming that off.'

(55) jái'si, [yy'tuu] yy'tia utajá'kwynajai'si ny'mikupa?juna nomiča'si ja. yaisi $\ddot{i i}=$ tia $\quad \mathrm{u}=$ taya $-\mathrm{kwinai}=$ yaisi

then you $=$ thusly $3 \mathrm{SG}: \mathrm{ACC}=$ send-THROW: $\mathrm{SG}=$ then

nimmi-kuba-Pyu-na nomi-ča-si yaa

1PL.EXCL-upon-from-PTCP move-away-SEQ here

'But then (the younger sister said to the older sister): "When you sent him away, you moved away from us here.",

(56) [nýa] nyy akumáka?ju kai yy míkajai'si katyha’máa ni’tá'ma.

nii a=guma-ga-?yu kai ii mii=ga=yaisi

I $4=$ husband-have-NOM NEG you $\quad$ QUOT $=$ THUS $=$ then

$\mathrm{ka}=\mathrm{t} \mathrm{i}=$ hamma?a nitama

$\mathrm{ACC}=\mathrm{LOGO}=$ elder.sister tell

“"I married him, not you," she told her older sister.'

(57) uusa'pa suha'máa hi'kwa'na manai tywa.

$\mathrm{uu}=$ sapa $\mathrm{su}=$ hamma $\mathrm{a}$ hikwa - nna manai tiwa

like.so=MOD NOM=elder.sister ??-PTCP happen also

'Thus the older sister was out of luck again.'

(58) o nataja?

oo na-taya

DEM MID-send

Listener: 'Then she was sent away?' 
(59) o natajau.

oo na-taya-u

DEM MID-send-PFV

'Then she was sent away.'

(60) oosu naa'ci?i, oo'kaa tuPi hii'sa'paka u'ka utymáca?i ú'ni'ku hii'pi kapoo.

$$
\begin{aligned}
& \mathrm{oo}=\mathrm{su} \quad \text { naatsi } \mathrm{Pi} \quad \mathrm{oo}=\mathrm{kaa} \quad \mathrm{tu} \mathrm{Pi}=\mathrm{hii}=\mathrm{sapa}=\mathrm{ga} \\
& \mathrm{DEM}=\mathrm{EMPH} \text { boy } \quad \mathrm{DEM}=\mathrm{ACC} \quad \text { any }=\text { thing }=\mathrm{MOD}=\mathrm{MOD} \\
& \mathrm{u}-\mathrm{ka} \quad \mathrm{u}=\text { timadza? } \mathrm{uuni}-\mathrm{ku} \text { hiipi } \\
& \text { 3SG-ACC 3SG:ACC =help.TR that.kind-ACC whatever } \\
& \mathrm{ka}=\text { ?oo } \\
& \mathrm{ACC}=\mathrm{DEM}
\end{aligned}
$$

'The boy there, I wonder what(ever) it was that helped him (to get) that kind of possessions.'

(61) ty’tyha y?na?wi tu?i mítia ka'sunámina hii'sa'pa u'kau uhiima'ka.

titiha i?na?wi tu?i mii=tia ka=sunami-na

sorrow express try QUOT=thusly $\mathrm{ACC}=$ think:SG-PTCP

$$
\begin{array}{lll}
\text { hii =sapa } & \mathrm{u}-\mathrm{ka}-\mathrm{u} & \mathrm{u}=\text { hii-maka } \\
\text { thing=MOD } & \text { 3sG-ACC-FOC } & \text { 3SG:ACC=thing-give/feed }
\end{array}
$$

'He expressed the sorrow he felt for himself, and something gave him those things.'

(62) kai nasó' pita'kwa'tu.

kai na-sopitakwatu

NEG MID-understand

'It is inconceivable.'

(63) jakana miu y?na?wi.

$\begin{array}{lll}\text { yaga-na } & \text { mi-u } & \text { iPna?wi } \\ \text { cry-PTCP } & \text { QUOT-FOC } & \text { express }\end{array}$

Listener: 'He cried and said (something),'

(64) uu'su hii nanasu'kwykai symytui manai'titia?a.

$\mathrm{uu}=\mathrm{su} \quad$ hii nanasukwigai $\operatorname{simi}=$ tui manaiti=tia?a

like.so $=\mathrm{EMPH}$ thing miraculous one $=$ any happen $=$ thusly

'Whatever it happens to be, it is miraculous, (but) once in a great while it might happen.'

(65) oo'su nanasu'kwykaity mi nani'tamaty.

$\mathrm{oo}=\mathrm{su}$ nanasukwigai-di mii na-nitama-di

DEM=EMPH miracle-NMLZ QUOT MID-tell-NMLZ

'That is the miracle talked about.' 
(66) nymy 'naa ny'my tymacaihuna u'suuka ka?oo.

nimi naa nimmi timadzai-hu-na $\mathrm{u}-\mathrm{su}=\mathrm{ga}$

person father people help-PFV-PTCP 3SG-NOM=MOD

$$
\begin{aligned}
& \mathrm{ka}=\text { ?oo } \\
& \mathrm{ACC}=\mathrm{DEM}
\end{aligned}
$$

'It is God who helps people; perhaps that is it.'

(67) kai haka so'pita'kwatuna.

kai haga sopitakwatu-na

NEG who understand-PTCP

'Nobody knows what (it is).'

(68) uu'su hii sunatýkwinapa ma'manimi?a.
$\mathrm{uu}=\mathrm{su}$
hii $\quad$ su=na-dikwinaba
ma mani-mi?a

like.so $=\mathrm{EMPH}$ thing $\mathrm{NOM}=\mathrm{MID}-$ recount DISTR $\sim \mathrm{do}-\mathrm{go}$

'That is something the stories tell about, and that happens sometimes.'

(69) ó’nojai'si sunymýpyy, kahi’má nawaha kai uni?óiču'ky’ti.

oono $=$ yaisi $\quad \mathrm{su}=$ nimi $=$ bii $\quad \mathrm{ka}=$ himma na-waha

at.the $\cdot$ time $=$ then $\quad \mathrm{NOM}=$ person $=\mathrm{FOC} \quad \mathrm{ACC}=$ what $\quad$ MID-tell

kai $\mathrm{u}=$ ni-?oiču-ki-ti

NEG 3SG:ACC =IP/SPEECH-believe-APPL-TNS

'And yet, the people, when something is told, they do not believe it.'

(70) kai uni?óiču'kyna jai'si, sunymýsa'kwa ka?uu tapya, waha'ma kwynyna waahu tuu py'takaana pui'kaana woo'kaa, mi nani' tamapaana o?uu tapy?a sunymý.

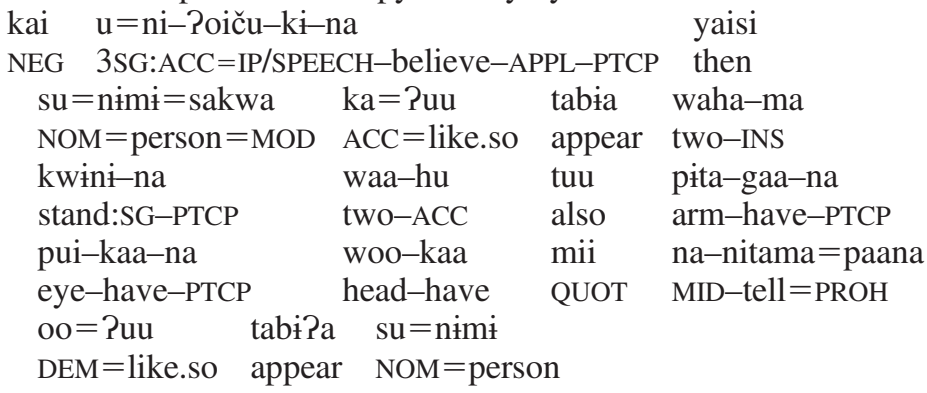

'They would not believe it, but man should look like this: stand with two (legs) and just have two arms, and have eyes, and have a head; that is what was told and that is the only way a person looks.'

(71) u'tuku mi?a onaatykwina'tu.

utugu mira o=naa-tikwina-tu

that.direction go:DUR 3SG:ACC $=$ MID-recount-ALL

'He goes through the tales.' 
(72) u’tuku miana ka?uu tapy?a, una?ýni'pywa?ni.

utugu mia-na ka=?uu tabipa

that.direction go-PTCP ACC=like.so appear:DUR

$\mathrm{u}=$ na-Pini-pi-wa?ni

3SG:ACC $=$ MID-Say-PERF-SIMIL

'He is going through it, and therefore he looks as it was said to be.'

(73) na'na'sytatywau ta'tapya'ku míha?a.

na na-sita = tiwau ta tabia-ku mii=ha?a

DISTR $\sim$ MID-bad $=$ also $\quad$ DISTR $\sim$ appear-PROSP $\quad$ QUOT $=$ QU

Listener: 'Are they going to look worse than each other?'

(74) jai'si, ó?nokajai'si kamiu.

yaisi óo?no $=\mathrm{ga}=$ yaisi $\quad \mathrm{ka}=\mathrm{mi}-\mathrm{u}$

then long.ago $=\mathrm{MOD}=$ then $\mathrm{ACC}=\mathrm{QUOT}-\mathrm{FOC}$

'And then . . . once upon a time (somebody) said it.'

(75) sunymý tui'kaa uni?óiču'ky’paana oo u'tuku mi?a upuní’paana kai uni?óiču'ky'ti py'mi tytyy'kwi?ja'pa tywau kai uni?óiču'ky'ti mi. $\mathrm{su}=\mathrm{nimi}=$ tui $=\mathrm{kaa} \quad \mathrm{u}=\mathrm{ni}-$ Poiču $-\mathrm{ki}=$ paana

$\mathrm{NOM}=$ person $=$ any $=\mathrm{MOD} \quad 3 \mathrm{SG}: \mathrm{ACC}=\mathrm{IP} / \mathrm{SPEECH}-$ believe $-\mathrm{APPL}=\mathrm{PROH}$

oo utugu mi?a $\mathrm{u}=$ puni $=$ paana

DEM that.direction go:DUR 3SG:ACC $=\mathrm{See}=\mathrm{PROH}$

kai $\mathrm{u}=$ ni-Póiču-ki-ti pimmi

NEG 3SG:ACC $=$ IP/SPEECH-believe-APPL-TNS they

ti $\sim$ diikwi $=$ ?yapa

DISTR $\sim$ tell $=$ PTC

tiwau kai $\mathrm{u}=$ ni-Poiču-ki-ti mii

also NEG 3SG:ACC=IP/SPEECH-believe-APPL-TNS QUOT

'Even though a person does not believe it, there he (nevertheless has to) go through it; (but) even though he sees it, he does not believe it; even though he is told, again he does not believe it, it is said.'

(76) mi sunymý nani'ta'ma.

mii su=nimi na-nitamma

QUOT NOM=person MID-tell

'That is what is told about man.'

(77) jai'si, kahi'má hau mani'si tuu (kam) oo nymywa?nisu mani'kwy'sia'pa kai uni?óiču'ky'ti usu'píta'kwatu' paana mí tywau.

yaisi $\mathrm{ka}=$ himma hau mani-si tuu

then $\mathrm{ACC}=$ what hau do-SEQ also

oo nimi-wa?ni-su mani-kwi-si=apa

DEM person-SIMIL-ADV do-FUT-SEQ=MOD 


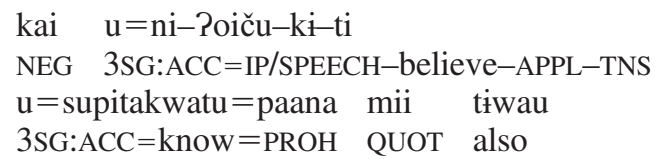

'And then, when something really happens, even though they will act as other people, they do not believe it, even though they do know it.'

(78) kaijai'si hii ka?u'káu natyykwinapa 'nawaha'na.

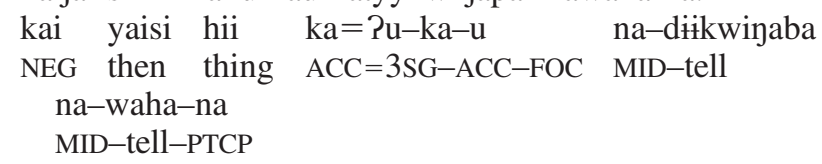

'There is nothing more than what is told in the tales.'

(79) kai haka ukau uuna'kwa maniwa?ni?ju.

kai haga $\mathrm{u}-\mathrm{ka}-\mathrm{u} \quad \mathrm{uu}=$ nakwa mani-wa?ni-Pyu

NEG who 3SG-ACC-FOC like.so=beside do-SIMIL-NOM

'Nothing can surpass them.'

(80) uuni?ju.

uuni-Pyu

that.kind-NOM

'That is how it is.'

(81) oo'kaa múPa'su ka?u'suu natyykwinapa su?i'sá ka?icáPanoo.

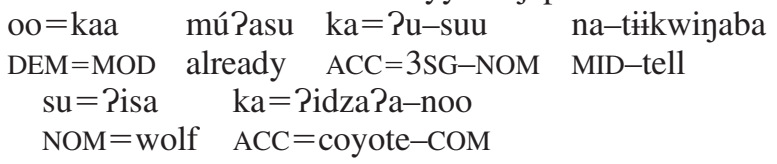

'That was the stories of the early days, (about) Wolf and Coyote.'

(82) kahi'máa miu.

$\mathrm{ka}=$ himmaa $\mathrm{mi}-\mathrm{u}$

$\mathrm{ACC}=$ what $\quad \mathrm{QUOT}-\mathrm{FOC}$

'Whatever they said.'

(83) suhii nani'nawamina nani'nawamina.

su $=$ hii $\quad$ na-ni-nna-wami-na

NOM=thing $\quad$ MID-IP/SPEECH-PTCP-PROG:DU-PTCP

na-ni-nna-wami-na

MID-IP/SPEECH-PTCP-PROG:DU-PTCP

'Anything that is talked and talked about.'

(84) nani'mama'kumina mí, nani'mama'kwymina mí, noo?juna nani'namuapi'tykaa'si, nani'mama'ku'si jai'si tuu, o namama'ku'py mi, noo?juna hii namama'ku'py hii uu mani'pyni'ku mii na?yni'si mi. 


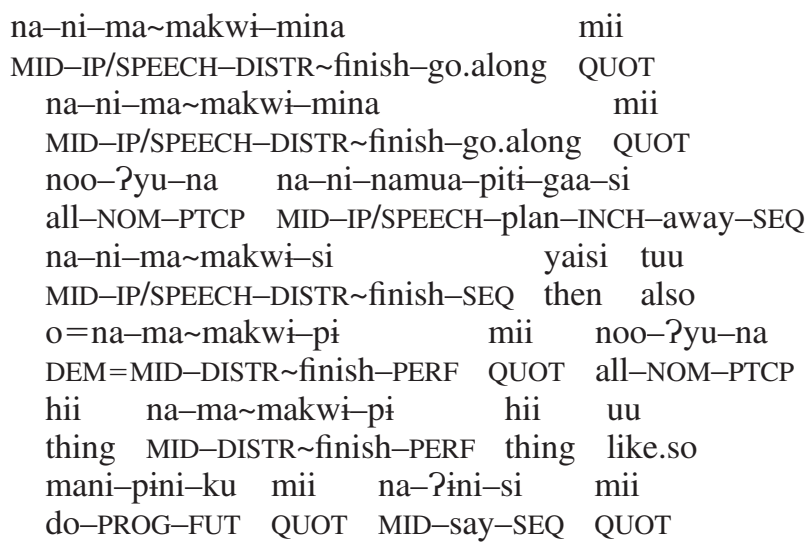

'Little by little things will get settled, it is said, things will get settled; and after they have talked about everything and finally finished everything, then it will be completed, it is said, everything of all kinds will be completed; that is how it will be as it was said.'

(85) oo u'tuku'su hii mi?a.

oo utugu-su hii mi?a

DEM that.direction-ADV thing go:DUR

'That is what we are going through.'

(86) oono'tu u'suu.

oono-tu u-su-u

at.the.time-LOC $3 \mathrm{SG}-\mathrm{NOM}-\mathrm{FOC}$

'That is the end of it.'

\section{THE FLOOD AND FIRE MYTH ${ }^{3}$}

Pete Snapp, Narrator

(1) mymu?a'su nymy, kamuika'su tii' pywai?juna, nana?a'kwina u ma?na?wi.

$$
\begin{aligned}
& \mathrm{mi}=\text { mu } \text { Pasu nimi } \quad \mathrm{ka}=\text { mui-ga-su tiipi-wai-Pyu-na } \\
& \mathrm{PL}=\text { already person } \mathrm{ACC}=\text { lead-away-ADV earth-AREA-from-PTCP } \\
& \text { na na-Pakwi-na uu maPna?wi } \\
& \text { DISTR MID-shoot-PTCP like.so do/act }
\end{aligned}
$$

'The first people, who were on the earth, fought each other, so they did.'

(2) nana'ky'sičana u ma?na?wina, jai'si, u'su my'naajai'si kai kaitia kai upicápina.

$$
\begin{array}{llll}
\text { na na-kisiča-na } & \text { uu } & \text { maPnaPwi-na } & \text { yaisi } \\
\text { DISTR MID-fight.against-PTCP } & \text { like.so } & \text { do/act-PTCP } & \text { then }
\end{array}
$$

\footnotetext{
${ }^{3}$ Recorded by Sven Liljeblad in 1962, Fort McDermitt Indian Reservation.
} 


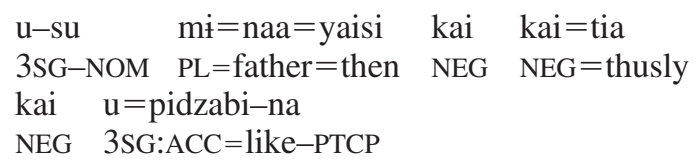

'They were busy fighting each other, but then their Father did not like it, not at all.'

(3) óčai'si ky'ma?asu manymytu'ku mi ynina.

$\mathrm{oo}=$ čaisi kimma?a-su ma-nimi-tu-ku mii

$\mathrm{DEM}=$ then different-ADV IP/HAND-person-make-FUT QUOT

ini-na

say-PTCP

'So then he said: "I will create another (world).",

(4) jai'si, hausa'kwa nyy, i'katíi'py sa'kwanýy hau atynái mítia?a.

yaisi hau $=$ sakwa nii $\mathrm{i}-\mathrm{ka} \quad$ tiipi $=s a k w a=$ nii

then how $=$ MOD I PROX - ACC earth $=$ MOD $=$ I

hau a=tinai $\mathrm{mii}=$ tia?a

how $4=$ burn $\quad$ QUOT=thusly

'And so, "I wonder what I should do, should I burn the earth?" he said.'

(5) tywausa'kwa hau nyy apa'tá'co’pa'ky míka.

tiwa-u=sakwa hau nit a=ba-ta-tsopa-ki

also-FOC $=$ MOD how I $4=$ IP/WATER-IP/FOOT-sink-APPL

$\mathrm{mii}=\mathrm{ga}$

QUOT=thusly

“"Or, rather, should I drown it?" he said.'

(6) mi yni'si jai'si, óčai'si apa'tá'co'pa'ky katii'py.

mii ini-si yaisi oo čaisi

QUOT say-SEQ then DEM then

$\mathrm{a}=$ ba-ta-tsopa-ki $\quad \mathrm{ka}=$ tiipi

$4=$ IP/WATER - IP/FOOT - sink - APPL $\quad$ ACC $=$ earth

'That was what he said, and then he drowned it, the earth.'

(7) [kai] kai atyná?itia?a.

kai kai $a=$ tina $i=$ tia?a.

NEG NEG $4=$ burn=thusly

'He did not burn it.'

(8) o'noo?o'ko mynymý'no'ko.

onnoo?o-ko mi=nimi-nno-ko.

entire-ACC $\quad \mathrm{PL}=$ person-all-ACC

Listener: 'All of it including the people?' 
(9) o?oi'kua'pa.

o?oikuapa.

everything

'Everything.'

(10) i'sujai'si paumana u manina paumana u maninajai'si isutíi'py pa'ta'co'paka?a.

i-su=yaisi pauma-na uu mani-na pauma-na

$\mathrm{PROX}-\mathrm{NOM}=$ then rain $-\mathrm{PTCP}$ like.so do-PTCP rain-PTCP

uu mani-na=yaisi i-su tiipi

like.so do-PTCP $=$ then $\mathrm{PROX}-\mathrm{NOM}$ earth

pa-ta-tsopa-ga?a

IP/WATER-IP/FOOT-sink-away

'Then this (happened), it was raining and raining, and the earth was slowly going under water.'

(11) pa'ta'co'pakaana, opii noo?juna pata'copi'taupi'tykaa.

pa-ta-tsopa-gaa-na obii noo-?yu-na

IP/WATER-IP/FOOT-sink-away-PTCP DEM all-NOM-PTCP

pa-ta-tsopita-u-piti-gaa

IP/WATER-IP/FOOT-sink:PL-PFV-arrive-away

'It was slowly going under water one thing after the other, until everything was covered.'

(12) u'su hucičai'si, katii'py pata'copi'taupi'tykaa'si, kau'ká ko'pi'topy kwyymajai'si ka?óo ka?utýka'tyty 'kuty'kwai ja.

u-su hudzi $=$ čaisi $\mathrm{ka}=$ tiipi

$3 \mathrm{SG}-\mathrm{NOM} \quad$ sagehen $=$ then $\quad \mathrm{ACC}=$ earth

pa-da-tsobita-u-piti-gaa-si

WATER-FOOT-sink.PL-PFV-INCH-away-SEQ

$\mathrm{ka}=\mathrm{u}-\mathrm{ka} \quad$ kopitobi $\quad \mathrm{kwihi}-\mathrm{ma}=$ yaisi $\mathrm{ka}=\mathrm{oo}$

$\mathrm{ACC}=3 \mathrm{SG}-\mathrm{ACC}$ ember grab - on $=$ then $\quad \mathrm{ACC}=\mathrm{DEM}$

$\mathrm{ka}=$ utikatidi kudi-kwai yaa

$\mathrm{ACC}=$ tall.mountain top-AREA here

'But the Sagehen, when the earth gradually sank in the water, picked up a burning coal of fire somewhere (and brought it) here to the top of utyka'tyty Tall Mountain.'

(13) oo uta'máky’nana [uha'máha] uta'mánapina uta'máky’nata'pi.

oo $\mathrm{u}=$ tammakinna-na

DEM 3SG:ACC =hold.down-PTCP

$$
\begin{array}{ll}
\mathrm{u}=\text { tammanabi-na } & \mathrm{u}=\text { tammakinna-dapi } \\
\text { 3SG:ACC=hold.down-PTCP } & \text { 3SG:ACC=hold.down-lie.SPL }
\end{array}
$$

'There she held it down and was lying on it as she held it down.' 
(14) upuuhita'pi.

$\mathrm{u}=$ puuhi-dapi

3SG:ACC $=$ blow-lie.SPL

'She was lying and blowing on it.'

(15) upuuhitapina jai'si, oo u'su ó'no'kaa u'su jaa u'ku'tá 'na?u'na'kwai'ty [kanai] nai'pyka?ju ku' pi'ta'pytia?a.

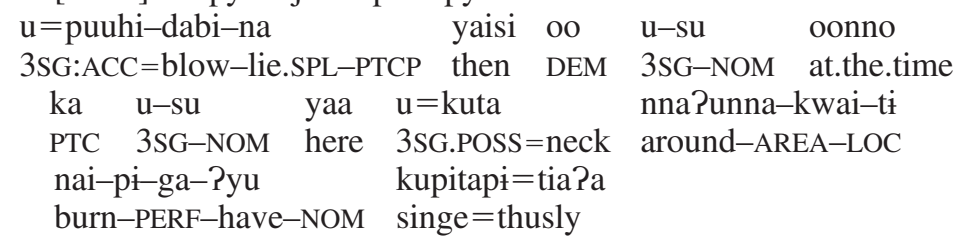

'And then, while she was lying and blowing on it, there it burnt right around her neck and burnt some (of the feathers).'

(16) oo uu u'su.

oo uu u-su

DEM like.so 3SG-NOM

'That is why she is like that.'

(17) oo uu ujykwína suhucíčai'si kako'sótia?a oo kai umatú'ki'kyna.

$$
\begin{aligned}
& \text { oo uu u=yikwi-na } \quad \mathrm{su}=\text { hudzi=čaisi } \\
& \text { DEM like.so 3SG:ACC }=\text { carry }-\mathrm{PTCP} \quad \mathrm{NOM}=\text { sagehen }=\text { then } \\
& \mathrm{ka}=\mathrm{kosso}=\text { tia?a } \quad \text { oo kai } \\
& \mathrm{ACC}=\mathrm{ash} / \mathrm{coal}=\text { thusly } \quad \mathrm{DEM} \quad \mathrm{NEG} \\
& \mathrm{u}=\text { ma-duki-ki-na } \\
& \text { 3SG:ACC }=\text { IP/HAND-extinguish-APPL-PTCP }
\end{aligned}
$$

'So in that way she took care of it, the sage hen; there (she took care of) the fire, so it wouldn't go out.'

(18) u'sujai'si ka?i'káu tii'py oi mani'si, u'kau sunymy' pi'ta.

$$
\begin{aligned}
& \mathrm{u}-\mathrm{su}=\text { yaisi } \mathrm{ka}=\mathrm{i}-\mathrm{ka}-\mathrm{u} \text { tiipi oi mani-si } \\
& 3 \mathrm{SG}-\mathrm{NOM}=\text { then } \mathrm{ACC}=\mathrm{PROX}-\mathrm{ACC}-\mathrm{FOC} \text { earth DEM do-SEQ } \\
& \mathrm{u}-\mathrm{ka}-\mathrm{u} \quad \mathrm{su}=\mathrm{nimi} \text { pita } \\
& \text { 3SG-ACC-FOC NOM=person build.fire }
\end{aligned}
$$

'And now, as this earth evolves, that is the thing people will make fire with.'

(19) u'su huci?ikaa'kaa, upa'tá'tu'ki'ky'si ikaa, i'kau tii'pykupa'ty ika

$$
\text { [katu?] katu?u napitaty. }
$$

$$
\begin{array}{ll}
\text { u-su } & \text { hudzi } 2 \mathrm{i}=\text { gaa }=\text { kaa } \\
\text { 3SG-NOM } & \text { sagehen }=\text { MOD }=\text { MOD } \\
\text { u=pa-ta-tuki-ki-si } \\
\text { 3SG:ACC = WATER-quench-APPL-SEQ }
\end{array}
$$




$$
\begin{array}{lll}
\text { ikaa } & \text { i-ka-u } & \text { tiipi-guba-ti } \\
\text { PROX-ACC } & \text { PROX-ACC-FOC } & \text { earth-upon-LOC } \\
\text { i-ka } & \text { kaduPu } & \text { na-bida-di } \\
\text { PROX-ACC } & \text { gone } & \text { MID-build.fire-NMLZ }
\end{array}
$$

'That sage hen, if she had let the fire go out, then there would have been no fire burning on this earth.'

(20) ika nymy sy'tyai'ku.

$\begin{array}{llll}\text { Oo } & \text { i-ka } & \text { nimi } & \text { sitiai-ku } \\ \text { DEM } & \text { PROX-ACC } & \text { person } & \text { freeze-PROSP }\end{array}$

Listener: 'Then people would freeze to death.'

(21) oo ha?ua'pakaa ika mynymy' ma'kupa'ty moo'tapya ikaa'kaa, u'ka ko'so katu?u'ku.

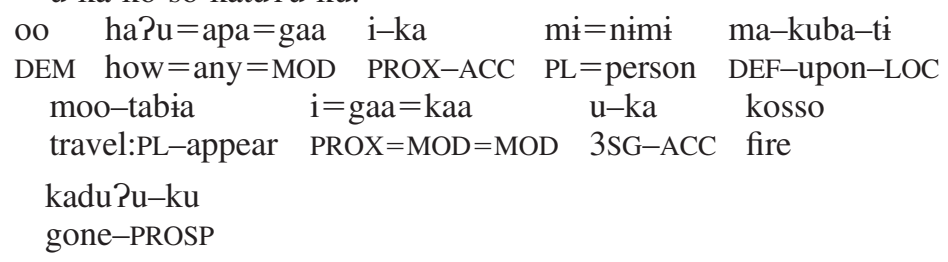

'I don't know how people would have gone about on it (on the earth) then without the fire.'

(22) o’uu uu namakico?ai'py soko'só o nymytia?a upi'tá.

$$
\begin{array}{llll}
\text { uu } & \text { na-ma-kidzo?ai-pi } & \text { su=kosso } & \text { oo } \\
\text { like.so } & \text { MID-IP/HAND-survive-PERF } & \text { NOM=coal } & \text { DEM } \\
\text { nimi }=\text { tia?a } & \mathrm{u}=\text { pita } & \\
\text { person = thusly } & 3 \mathrm{sG} \text { :ACC=build.fire:DUR }
\end{array}
$$

'That is how the fire was saved, and now people burn it.'

(23) wo?i'tu mi yni'si uma'ku pitau.

wo?itu mii ini-si u-ma-ku pida-u

firedrill QUOT say-SEQ 3SG-INS-LOC build.fire-PFV

'Through (use of) the firedrill hearth, as people call it, (somebody) made fire with it.'

(24) umatú'nuiwynyna soko'sótuau'si napitau.

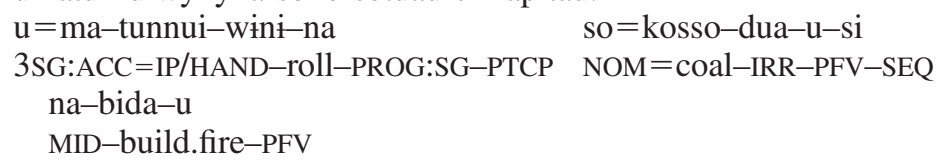

'He rolled it between his hands, and when sparks were formed fire was made.' 
(25) o?oho u'suu.

o?o u-Su-u

DEM 3SG-NOM-FOC

'That was it.'

(26) jai'si ó’nojai'si kamiu ka?i'ká tii'pykupa ny'my manymytu'si.
yaisi ónno=yaisi
$\mathrm{ka}=\mathrm{mi}-\mathrm{u}$
$\mathrm{ka}=\mathrm{i}-\mathrm{ka}$
then at the $\cdot$ time $=$ then $\quad \mathrm{ACC}=\mathrm{QUOT}-\mathrm{FOC} \quad \mathrm{ACC}=\mathrm{PROX}-\mathrm{ACC}$
tiipi-guba nimmi ma-nimi-du-si
earth-upon person IP/HAND-person-make-SEQ

'And then, when he created people on this earth he said (the following).'

(27) jau čuusa?a ka?iwí?juna nama?nawipy?wa?nisu, i'ka tii’pykupa'ty nama?na?wina.

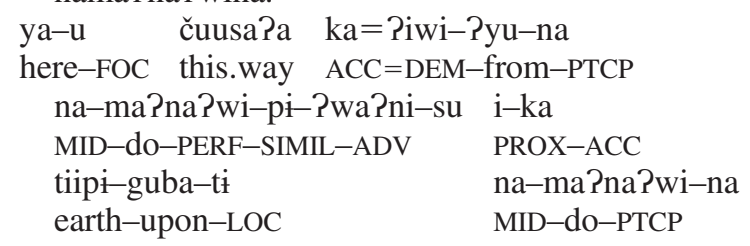

'From now on the same that was done here before will be done on this earth.'

(28) juutia?a pisa na'na'pypya?akaa.

yuu =tia?a pisa na nna-pi $\sim$ biaPa-kaa

this.way=thusly good DISTR $\sim$ MID-PL friend-have

'Be friendly to each other.'

(29) jai'sisaa katu?í hi'maatui matai mani'mina'kaa.

yaisi $=$ saa $\quad k a=$ tu $\mathrm{i}=$ himma $=$ tui madai mani-mmina $-\mathrm{kaa}$

then=later $\quad \mathrm{ACC}=$ any $=$ what $=$ any achieve do-go.along-away

'And then gradually they will learn how to make anything.'

(30) $[\mathrm{ka} ? \mathrm{~m}]$ u'my taipootia'kaa mai nymypaa mani'si mi, mai nymypaa pi'ty'si kapaa ?una'paamana'kwana.

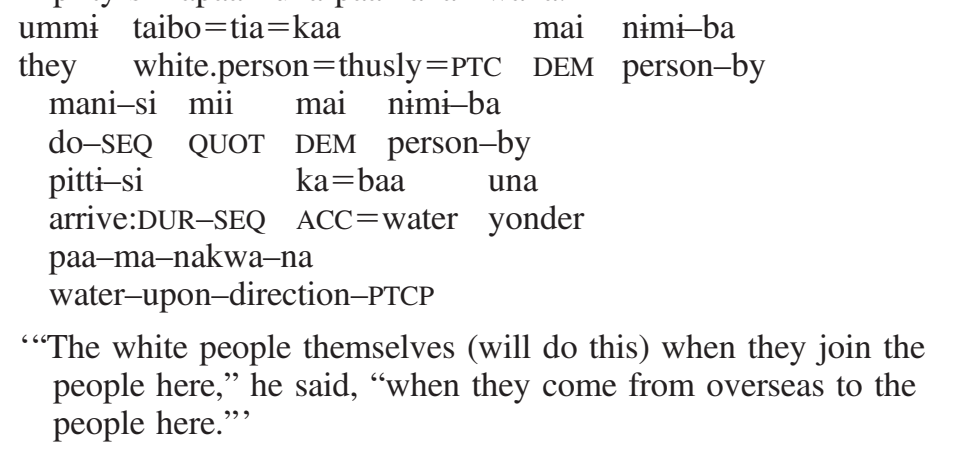


(31) saajai'si, pisa u'mynoo nahai'cikaana mytymácaina, pisa myma'týkyna u'mypaa?jutua.

saa $=$ yaisi pisa ummi-noo na-haitsi-kaa-na

later $=$ then good they-WITH MID-friend-have-PTCP

$\mathrm{mi}=$ timadzai - na $\quad$ pisa $\mathrm{mi}=$ ma-tigi - na

$\mathrm{PL}=$ help-PTCP $\quad$ good $\quad$ PL $=$ IP/HAND-put:SG-PTCP

ummi-ba-Pyu-dua

they-by-from-IRR

'And later on be good friends with them and help them, treat them well and unite with them.'

(32) kasaajai'si ka?u'káutia?a moí'ykaa'su tii'pykupa ni'matáimi'py,

u'kau noo?o'ko manamuamina mi kani'ni námuami'py mi.

$\begin{array}{lll}\mathrm{ka}=\mathrm{saa} & \text { yaisi } \mathrm{ka}=\mathrm{u}-\mathrm{ka}-\mathrm{u}=\text { tiaPa } & \text { moi'i-gaa-su } \\ \text { ACC=later then } & \mathrm{ACC}=3 \mathrm{SG}-\mathrm{ACC}-\mathrm{FOC}=\text { thusly } & \text { first-away-ADV } \\ \text { tiipi-guba } & \mathrm{ni}=\mathrm{mmadaimi}-\mathrm{pi} & \mathrm{u}-\mathrm{ka}-\mathrm{u} \\ \text { earth-upon } & \text { 1PL.EXCL:ACC=achieve-PERF } & \text { 3SG-ACC-FOC } \\ \text { noo?oko } & \text { manamua-mina } & \text { mii } \\ \text { all-ACC } & \text { do.repeatedly-go.and } & \text { QUOT } \\ \text { ka=ni=nni-namuami-pi } & \text { mii } \\ \text { ACC=1PL.EXCL:ACC -IP/SPEECH-happen-PERF } & \text { QUOT }\end{array}$

'And then later, what we brought about on the first earth, all that we have talked about, they (the people now) have to bring to pass.'

(33) u'kau noo?o'ko mataimina.

$\mathrm{u}-\mathrm{ka}-\mathrm{u}$ noo?o-ko madai-mina.

3SG-ACC-FOC all-ACC achieve-go.along

'All that they are (now) accomplishing little by little.'

(34) ynyysu tymáta?i mi nasu?jykwimina.

ini-su ti-mada?i mii na-su-Pyigwi-mina

INT-ADV ANTIP-achieve:DUR QUOT MID-IP/MIND-do-go.along

'They think of themselves as achieving a great deal.'

(35) nyy ynyy hi'ma so'pita'kwatuna.

nii ini himma sopidakwatu-na

I INT what know-PTCP

'I really know everything.'

(36) noo?o'ko hi'ma uuna'kwa nyy mani'mina mi nasu?jykwina.

noo?o-ko himma uu=nakwa nï mani-mmina

all-ACC what like.so=direction I do-go.along

mii na-su-Pyigwi-na

QUOT MID-IP/MIND-do-PTCP

'I can do anything better than anybody else," he thinks of himself.' 
(37) saajai'si tuu ka?unáa umacá'su Pamina, kawahámano'kwai'ty, ó?no'kwai'ty to'mo manipi'tykaa'si.

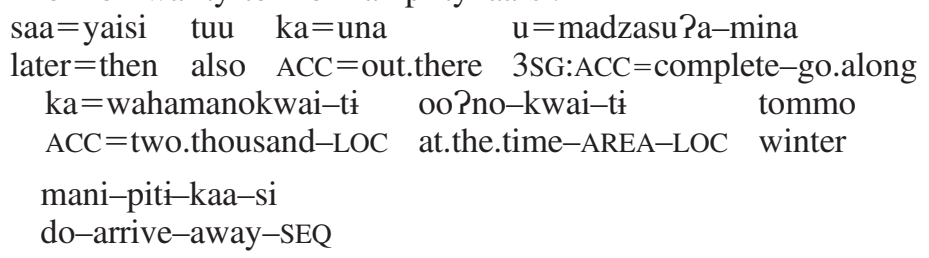

'And then finally it is about to be done, when it is getting close to (the end of) the two thousand years.'

(38) nyy syta mymanípi'tykaa'si ónosa?a tuu i'ka tii'py a'tasu jykwitua míkajai'si yná'kwi.

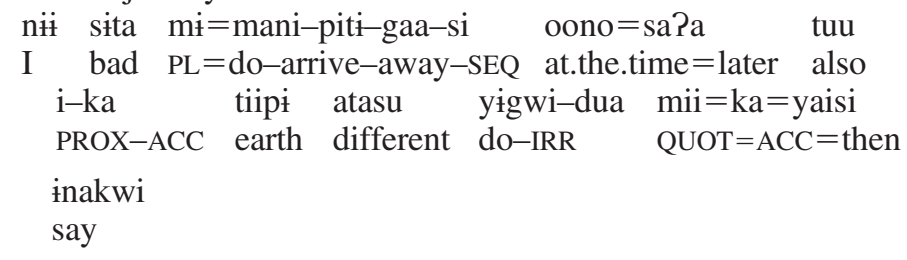

"When they get worse, then finally I will change this earth," that is what he said.'

(39) jai'si kaitiya?a mytyy'kwi nyysa?a, uu'saa nyy kaja ajykwítua mi kai yna'kwi.

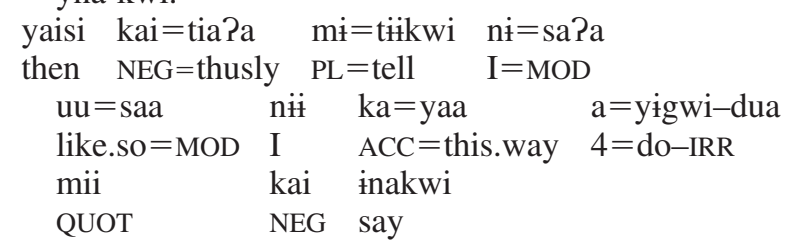

'But he did not tell them: "I shall treat it that way this time," that he did not say.'

(40) oo a'tasu nyy apytýčykwi'ku'su katii'py mí'sy?my yna'kwi mi.

$$
\begin{aligned}
& \text { oo atasu nii a=pidi-čikwi-ku-su } \\
& \text { DEM different I } 4=\text { recent-do-PROSP-ADV } \\
& \mathrm{ka}=\text { tiipi } \mathrm{mii}=\mathrm{si} \text { ?mi inakwi mii } \\
& \mathrm{ACC}=\text { earth } \quad \mathrm{QUOT}=\text { only say } \quad \text { QUOT }
\end{aligned}
$$

“"Then I will make the world over in a different way," was all he said.'

(41) mynymy’jai'si mi uni'sú'piča mymu?a'su nymyka kunatypinana.

$\mathrm{mi}=$ nimi $=$ yaisi mii $u=$ ni-supiča

$\mathrm{PL}=$ person $=$ then $\mathrm{QUOT} 3 \mathrm{SG}: \mathrm{ACC}=\mathrm{IP} / \mathrm{SPEECH}-$ consider 


$$
\begin{array}{lll}
\mathrm{mi}=\text { mú Pasu } & \text { nimi }=\mathrm{ga} & \mathrm{ku}=\text { na }- \text { dibina }- \text { na } \\
\mathrm{PL}=\text { already } & \text { person=MOD } & \mathrm{ACC}=\mathrm{MID}-\text { recount }-\mathrm{PTCP}
\end{array}
$$

'People then wonder about it, as the old time people told about it.'

(42) ha?ukainasa?a ajykwítua'ti.

ha?u gaina $=$ sa?a a=yigwi-dua-ti

how $\quad$ INFER $=$ later $4=$ do-IRR - TNS

'What is he going to do with it later?'

(43) kamui?yka'su pyy oo atynái'ku mi tu?i yni'si jai'si kai atynáina apa'tá'co'pa'ky miitia?a.

$$
\begin{array}{lllll}
\text { ka=muiPiga-su=bii } & \text { oo } & \text { a=tinai-ku } & \text { mii } & \text { tuPi } \\
\text { ACC =first-ADV=as.for DEM } & 4=\text { burn-PROSP } & \text { QUOT } & \text { try } \\
\text { ini-si } & \text { yaisi } & \text { kai } & \text { a=tinai-na } \\
\text { say-SEQ } & \text { then } & \text { NEG } & 4=\text { burn-PTCP } \\
\text { a=patatsopa-ki } & \text { mii=tiaPa } & &
\end{array}
$$

'First he said that he was going to burn it, that is what he tried to say; but he did not burn it; it was put under water, it is said.'

(44) já'ty'kwaikasa?a ha?uu.

Yaa ti-kwai $=\mathrm{ka}=\mathrm{saPa} \quad \mathrm{haP}=\mathrm{uu}$

here $\mathrm{ANTIP}-\mathrm{AREA}=\mathrm{ACC}=$ later $\mathrm{QU}=$ like.so

'How will it be this time?'

(45) ha?uukasa'a uu'su ajykwítua py’tyjá'tykwaikaa pyy atynái'ku mi u'my asy'kwíni'su'piča.

$$
\begin{array}{ccl}
\text { ha } \mathrm{Puu}=\mathrm{ga}=\mathrm{sa} \text { Pa } & \mathrm{uu}=\mathrm{su} & \mathrm{a}=\text { yigwi-dua } \\
\text { how }=\mathrm{MOD}=\mathrm{MOD} & \text { like.so=EMPH } & 4=\text { do-IRR } \\
\text { pi=tiyati-kwai=gaa } & \text { pii } & \mathrm{a}=\text { tinai-ku } \\
\text { PRO=??-AREA=MOD } & \text { RESTR } & 4=\text { burn-PROSP } \\
\text { mii } & \text { ummi } & \mathrm{a}=\text { sikwi-ni-supiča } \\
\text { QUOT } & \text { they } & 4=\text { only-IP/SPEECH-want }
\end{array}
$$

" "I wonder if he is going to do the same with it, or if it is this time he is going to burn it," that is what they guess.'

(46) ó'tia já'ty'kwai u'su atynái'si katujai'si hii mani'ku.

$$
\begin{array}{llll}
\text { oo=tia } & \text { yaa-ti-kwai } \quad \text { u-su } & \text { a=tinai-si } \\
\text { DEM=thusly } & \text { here-LOC-AREA } & \text { 3SG-NOM } & 4=\text { burn-SEQ } \\
\text { kaduPu=yaisi } & \text { hii mani-ku } & \\
\text { gone=then } & \text { thing } & \text { do-PROSP } &
\end{array}
$$

Listener: 'And if he burns it this time, will he destroy everything?'

(47) oo, oo?no'to u'suu naatywi?ya.

$$
\begin{array}{llll}
\text { oo } & \text { oo?no-to } & \text { u-su } & \text { naatiwi?ya } \\
\text { DEM } & \text { long.ago-LOC } & \text { 3SG-NOM } & \text { story }
\end{array}
$$

'That is as far as the story goes.' 